\title{
Close-range hyperspectral spectroscopy reveals leaf water content dynamics
}

\author{
Junttila, S. ${ }^{1}$, Hölttä, T. ${ }^{2}$, Saarinen, N. ${ }^{1}$, Kankare V. ${ }^{1}$, Yrttimaa T. ${ }^{1}$, Hyyppä, J. ${ }^{3} \&$ Vastaranta,
} M. ${ }^{1}$

${ }^{1}$ School of Forest Sciences, University of Eastern Finland, Joensuu, 80101, Finland

${ }^{2}$ Department of Forest Sciences, University of Helsinki, Helsinki, 00014, Finland

${ }^{3}$ Department of Remote Sensing and Photogrammetry, Finnish Geospatial Research Institute, National Land Survey of Finland (NLS), Masala, 02431, Finland

*Corresponding author at: School of Forest Sciences, University of Eastern Finland, Yliopistokatu 7, 80101 Joensuu, Finland. E-mail address: samuli.junttila@uef.fi (S. Junttila).

Keywords: leaf water content; hyperspectral spectroscopy; leaf water potential; drought; diurnal cycle; plant water status; relative water content; equivalent water thickness; Dracaena marginate; water stress; leaf water variation

\section{Research highlights}

- The sensors captured up to $96 \%$ of the variation in equivalent water thickness

- A method was developed for measuring diurnal changes in leaf water content

- Continuous measurements revealed detailed diurnal variation of leaf water content

\begin{abstract}
Water plays a crucial role in maintaining plant functionality and drives many ecophysiological processes. The distribution of water resources is in a continuous change due to global warming affecting the productivity of ecosystems around the globe, but there is a lack of non-destructive methods capable of continuous monitoring of plant and leaf water content that would help us in understanding the consequences of the redistribution of water. We studied the utilization of novel small hyperspectral sensors in the 1350-2450 nm spectral range in non-destructive estimation of leaf water content in laboratory and field conditions. We found that the sensors captured up to $96 \%$ of the variation in equivalent water thickness (EWT, $\mathrm{g} / \mathrm{m}^{2}$ ) and up to $90 \%$ of the variation in relative water content (RWC). These laboratory findings were supported by field measurements, where repeated leaf spectra measurements were in good agreement
\end{abstract}


$\left(R^{2}=0.79\right)$ with a time-lagged change of tree xylem diameter. Further tests were done with an indoor plant (Dracaena marginate Lem.) by continuously measuring leaf spectra while drought conditions developed, which revealed detailed diurnal dynamics of leaf water content. We conclude that close-range hyperspectral spectroscopy can provide a novel tool for continuous measurement of leaf water content at the single leaf level and help us to better understand plant responses to varying environmental conditions.

\section{Introduction}

Insights into the movement of water within ecosystems are crucial for understanding the responses of ecosystems to the changing climate. To monitor changes caused by altered water availability to vegetation, measurements of leaf and canopy water content are needed over varied spatial and temporal scales ranging from single leaves to landscapes and from diurnal to seasonal to assess plant water status (Konings et al., 2019; Rao et al., 2019). Droughts are causing limited availability of water for trees, and have been identified as one of the main drivers of increasing global tree mortality (Allen et al., 2010). Prior to mortality, limited water availability is also causing tree water stress and it manifests as a lower leaf water content. Leaf water content affects many metabolic processes such as stomatal conductance, photosynthesis and growth, but there is a lack of methods that are capable of continuously monitoring leaf water content of single leaves or tree canopies. Monitoring of tree canopy water content may lead to better understanding of the degree of tree water stress in forests (Allen et al., 2010; Williams et al., 2013).

Leaf water content can be assessed using multiple metrics. The direct physical representation of the layer of water within a leaf is measured as equivalent water thickness (EWT) or the mass of water per leaf area. EWT is often used as a metric in remote sensing literature due to the direct physical relationship between leaf absorption and EWT (Feret et al., 2019). However, EWT does not directly translate into plant water status, which we often are interested in. Leaf water potential is probably the most widely used metric of plant water status as it is strongly related to the relative water content (RWC) of leaves (i.e., the amount of water in the leaf per the amount of water in the leaf in full saturation). But due to the close relation between leaf spectra and EWT, remote sensing scientists have focused more on estimating EWT. However, the change in EWT can be linked to the change in RWC, which provides a possibility to estimate plant water status using leaf spectral reflectance. 
Remote sensing scientists have been studying the estimation of leaf water content for decades using the changes in infrared reflectance caused by altered leaf water content (Ceccato et al., 2001; Cheng et al., 2011; Danson et al., 1992; Penuelas et al., 1993; Penuelas et al., 1997; Tucker, 1980). There is a vast amount of research on this topic and it is not in the scope of this paper to go into details, but to give the reader an estimate, we searched the Web of Science for articles that had "leaf water content" and "remote sensing" as keywords and found 163 articles. Various approaches and techniques have been used with different application perspectives and varying level of success, for estimating leaf water content with wide ranging spatial scales, including terrestrial lidar (Elsherif et al., 2019a; Junttila et al., 2019; Junttila et al., 2021), imaging spectroscopy (Kotz et al., 2004), terahertz radiation spectroscopy (Browne et al., 2020) and microwave remote sensing (Konings et al., 2019). However, considering leaf water content monitoring from single trees several times a day (which is required for monitoring diurnal differences in leaf water content) there are still many limiting factors with existing remote sensing methodologies. The limitation of passive spectroscopy is its dependence on external illumination, challenging the collection of dense time-series for assessing diurnal leaf water content dynamics. Active sensors such as terrestrial lidar can provide backscatter measurements any time of the day, but these devices have not been designed for spectral measurements and suffer from calibration issues (Junttila et al., 2019; Korpela, 2017). In addition, the temporal resolution of many of the developed approaches has been limited. Repetition rate of the measurements in addition to illumination conditions (especially when using passive imaging) limit the temporal coverage of airborne and satellite measurements, again hampering the measurement of diurnal leaf water content dynamics. There has been a lack of methods that can accurately measure changes in leaf water content non-destructively with a temporal resolution of less than a minute. Respectively, widely applied methods used to assess plant water status, such as destructive measurements of RWC and leaf water potential, are labour intensive, require destructive sampling and are practically impossible to conduct at short time intervals for a long period of time (e.g. Turner and Long (1980)).

The advancement of sensor technology has recently enabled the construction of handheld spectrometers (Beć et al., 2020). Miniaturization and lower costs have opened up new avenues for scientific and practical applications using spectroscopy when the sensors are becoming more widely available both in research and business (Huck, 2021). Where a traditional spectrometer has required a backpack, the most recent sensors are the size of a matchbox and enable high temporal resolutions with continuous measurements of a single plant or a leaf, which are not 
possible using destructive sampling. In this study, we aimed to test if a small low-cost hyperspectral sensor could be used to monitor the diurnal dynamics of leaf water content, and potentially replace the use of destructive sampling methods in assessing leaf water content and plant water status.

The aim of the study was to evaluate the capabilities of low-cost hyperspectral spectroscopy in monitoring diurnal variation of leaf water content using both destructive and in-situ measurements. We had the following research questions (RQ):

RQ 1. Can hyperspectral spectroscopy in the spectral range between $1350 \mathrm{~nm}$ and $2450 \mathrm{~nm}$ be used to monitor the diurnal variation in leaf water content?

RQ 2. What kind of accuracy can be achieved in estimating leaf water content using low-cost hyperspectral spectroscopy?

These two research questions were investigated in three different experiments to estimate the accuracy of the developed method and to cover short- and long-term monitoring of leaf water content in both field and laboratory conditions.

\section{Material and methods}

\section{Investigated hyperspectral sensors}

We investigated three small sized hyperspectral sensors in leaf water content monitoring: NIRONE S1.7 (Spectral Engines, Espoo, Finland), NIRONE S2.0 and NIRONE S2.5. These sensors measure $1350-1650 \mathrm{~nm}$ region, $1550-1950 \mathrm{~nm}$ region and 2000-2450 $\mathrm{nm}$ region, respectively. The sensors have two vacuum tungsten lamps that illuminate the target. The wavelengths can be programmed but the full width at half maximum is $13-17 \mathrm{~nm}$ for the $\mathrm{S} 1.7$ sensor, $15-21 \mathrm{~nm}$ for the S2.0 sensor and $18-28 \mathrm{~nm}$ for the S2.5 sensor. The detector in the sensors is a single element extended InGaAs type. Light enters the detector through a hole that is one millimeter in diameter. The viewing angle of the sensor is only 8 degrees; thus, resulting in a narrow measurement area when measuring at close-range. Signal-to-noise ratio is 11000 for the S1.7 sensor, 7000 for the S2.0 sensor and 1500 for the S2.5 sensor. The sensor dimensions are $25 \times 25 \times 17.5 \mathrm{~mm}$ and it weighs $15 \mathrm{~g}$. The sensor parameters used in the measurements were a point average of 100 and a scan average of three. 


\section{Experiment 1.}

The first experiment was conducted on the $26^{\text {th }}$ of August, 2020 in the Viikki campus area in Helsinki, Finland. Twigs of Silver birch (Betula pendula Roth) were detached from a young Silver birch tree (height: $3.5 \mathrm{~m}$, diameter at breast height $3 \mathrm{~cm}$ ) and put immediately under water for a re-cut to maintain water connectivity in the xylem. Leaves were then covered with aluminium foil and bagged with a plastic bag to allow full rehydration of the leaves for three hours. The twigs were then carried to the laboratory for further measurements.

A fully hydrated leaf was detached, and a leaf disc of $10 \mathrm{~mm}$ diameter was cut from it, weighted and the leaf reflectance and transmittance were measured from the abaxial side using NIRONE S1.7 and S2.5 sensors consequently. The remaining leaf was left to dry for 30-60 minutes and an additional disc or two were cut during that time. We measured 21 leaves in total using a total of 44 leaf discs, i.e. two discs per leaf were measured on average. The leaf discs were then merged under water for 12 hours to allow full hydration and weighted to measure turgid weight. The leaf discs were then dried in $60{ }^{\circ} \mathrm{C}$ for 48 hours until they reached a constant mass and the dry weight was measured.

Table 1. The mean, minimum, maximum and standard deviation of equivalent water thickness (EWT) and relative water content (RWC) of the measured leaf disc samples $(n=44)$.

\begin{tabular}{l|llll} 
& Mean & Minimum & Maximum & Standard deviation \\
\hline EWT (g/m²) & 85.5 & 54.7 & 114.6 & 16.5 \\
RWC (\%) & 73.6 & 44.3 & 95.3 & 14.5
\end{tabular}

\section{Experiment 2.}

A second experiment was conducted between the $31^{\text {st }}$ of August and the $1^{\text {st }}$ of September 2020 in Hyytiälä Forest Research Station in Juupajoki municipality in Southern Finland, where a station for measuring ecosystem-atmosphere relations (SMEAR II) is located. The test forest site was a 58-year-old Scots pine (Pinus sylvestris L.) stand with admixtures of Norway spruce (Picea abies (L.) Karst.), rowan (Sorbus aucuparia L.), European aspen (Populus tremula L.), and common juniper (Juniperus communis L.). We monitored a mature Silver birch (height: 18 
m, diameter at breast height: $25 \mathrm{~cm}$ ) for 22 hours using NIRONE S1.7 and NIRONE S2.5 sensors and a linear displacement transducer dendrometer ( $G$ series spring Push, $\mathrm{Vg} / 5 / \mathrm{s}$, Solartron Inc., West Sussex, UK) at $1.5 \mathrm{~m}$ height to measure diurnal changes in xylem diameter. The diurnal xylem diameter variation was used as a proxy measurement of changes in leaf water content due to its strong correlation with leaf water potential when using a time lag (Junttila et al., 2021). This has been confirmed on the site before and we used a 90-minute time lag (Junttila et al., 2021). Ten healthy leaves were randomly selected from the canopy for repeated measurements using the NIRONE sensors. A marker was used to outline the measurement area to avoid the influence of within leaf variation of leaf properties on the measurement. A single measurement of each leaf was conducted at six time points during the monitoring period representing different leaf water status stages based on the diameter of the tree xylem.

\section{Experiment 3.}

The third experiment was conducted in laboratory conditions with a common monocot house plant: Dracaena marginate Lem. The plant consisted of two stems growing in the same pot with heights of $1.2 \mathrm{~m}$ and $0.5 \mathrm{~m}$. The plant was subjected to natural light from a west facing window, but the plant was subjected to direct sunlight only a few hours per day. Three hyperspectral spectroscopy sensors were installed in the plant to continuously measure leaf reflectance: NIRONE S1.7 (1350-1650 nm), NIRONE S2.0 (1550-1950 nm) and NIRONE S2.5 (2000-2450 nm). Each sensor was mounted to measure a different leaf. The NIRONE S1.7 and S2.5 were measuring leaves of the taller stem and the S2.0 was measuring a leaf of the shorter stem. A custom 3D printed sensor mount was developed for this purpose, which kept the leaf and the sensor in place. Because the sensors generate heat, they were installed to measure at a $1.0 \mathrm{~cm}$ distance. The sensor mount design can be found from the supplementary files. After the installation of the sensors, the plant was left to adjust for three days.

The D. marginate was subjected to drought during the monitoring period by scheduling a sparse watering schedule. The plant was monitored from $19^{\text {th }}$ March to $8^{\text {th }}$ April and was watered twice during this period: on $25^{\text {th }}$ March 1.01 and $6^{\text {th }}$ April 0.51 . The plant was well-watered before the start of the monitoring period.

We investigated how the spectra of each sensor changed during the time between the $26^{\text {th }}$ March and April $5^{\text {th }}$ (the period without watering), during which drought slowly developed as the amount of water in the soil depleted. We used time from last watering (TLW) as a coarse proxy 
of leaf water content during the drought period to investigate the spectral dynamics recorded by the NIRONE sensors. We note that there are limitations in the relationship between TLW and leaf water content, but it is well-known that leaf water content depletes as drought intensifies (Egilla et al., 2005; Li-Ping et al., 2006; White et al., 1996).

\section{Spectral features}

Spectral features were calculated from the measured reflectance values to investigate the dependence between spectra and leaf water content metrics. The measurements of NIRONE sensors were combined for each leaf and a normalized ratio index (NRI) of each wavelength combination was calculated (Equation 1).

$$
N R I=\frac{\gamma_{1}-\gamma_{2}}{\gamma_{1}+\gamma_{2}}
$$

where $\gamma_{1}$ and $\gamma_{2}$ are the reflectance of each wavelength.

The spectral features included the mean measured reflectance of each wavelength and the NRI.

Daily metrics of leaf spectra were calculated in the drought experiment (Experiment 3) to describe the development of spectral features over time. The daily metrics were the mean and range of each spectral feature for each day during the monitoring period.

\section{Statistical analysis}

Linear regression models were developed between the spectral features and the leaf water content metrics to investigate their relations and capability to explain variation in leaf water content. Single spectral features were used as predictors of the leaf water content metrics. Linear regression was also used to model the relations of spectral features and diurnal variation in diameter. In the third experiment, regression models were developed between TLW and daily metrics of spectral features to evaluate their capability in capturing changes in leaf water content over time during drought conditions.

We used coefficient of determination $\left(R^{2}\right)$, root-mean-square-error (RMSE), normalized rootmean-square-error (NRMSE, RMSE divided by the mean of the observed values) and mean absolute error (MAE) to evaluate the goodness of the models in explaining variation in leaf 
water content using leave-one-out cross-validation. The top five of the spectral features for explaining the variation in leaf water content are depicted in the results for each experiment. All the statistical analyses were conducted using R software (R Core Team, 2013) and the hsdar package for convenient handling of spectral data.

\section{Results}

\section{Estimation of leaf water content using leaf spectra in the short-wave infrared (SWIR) region (Experiment 1)}

Both investigated sensors showed high sensitivity to changes in leaf water content with increasing reflectance indicating decreasing leaf water content. Reflectance of every wavelength in both investigated spectral regions showed their capacity of being capable of estimating EWT with accuracies (RMSE) ranging between $5.91 \mathrm{~g} / \mathrm{m}^{2}$ (NRMSE: $6.9 \%$ ) and 9.48 $\mathrm{g} / \mathrm{m}^{2}$ (NRMSE: 11.1\%) (Table 1). NIRONE S2.5 with the spectral range of 2000-2450 nm showed more consistent prediction accuracies of both EWT and RWC compared to the 1350$1650 \mathrm{~nm}$ range. The most accurate models in estimating EWT were using wavelengths: 2020 $\mathrm{nm}, 2050 \mathrm{~nm}, 2010 \mathrm{~nm}$, and $2030 \mathrm{~nm}$. The most accurate models in estimating RWC were developed using wavelengths: $2010 \mathrm{~nm}, 2020 \mathrm{~nm}, 2000 \mathrm{~nm}$, and $2040 \mathrm{~nm}$. However, there were small differences ( $R^{2}$ varied only 0.03 units) between wavelengths in the 2000-2450 nm domain in the estimation accuracy of both leaf water content metrics.

Table 2. Statistics of linear regression models between each wavelength, measured using NIRONE S1.7 and NIRONE S2.5 sensors, and equivalent water thickness (EWT, g/m²) and relative water content (RWC). Coefficient of determination $\left(R^{2}\right)$, root-mean-square-error (RMSE) and mean absolute error (MAE) were calculated using single wavelength features ( $n=44)$. Mean, maximum (max), minimum (min) and standard deviation (std) are calculated from all the developed regression model statistics.

\section{EWT}

\begin{tabular}{|c|c|c|c|c|c|c|c|c|c|}
\hline \multicolumn{5}{|c|}{ NIRONE S1.7 1350-1650 nm } & \multicolumn{5}{|c|}{ NIRONE S2.5 2000-2450 nm } \\
\hline & mean & $\max$ & $\min$ & std & & mean & $\max$ & $\min$ & std \\
\hline$R^{2}$ & 0.76 & 0.80 & 0.66 & 0.39 & $\boldsymbol{R}^{2}$ & 0.86 & 0.87 & 0.86 & 0.004 \\
\hline
\end{tabular}




\begin{tabular}{|c|c|c|c|c|c|c|c|c|c|}
\hline RMSE & 8.0 & 9.48 & 7.29 & 0.63 & RMSE & 6.07 & 6.21 & 5.91 & 0.09 \\
\hline NRMSE & $9.3 \%$ & $11.1 \%$ & $8.5 \%$ & $0.7 \%$ & NRMSE & $7.1 \%$ & $7.3 \%$ & $6.9 \%$ & $0.1 \%$ \\
\hline MAE & 6.46 & 7.69 & 5.86 & 0.52 & MAE & 4.75 & 4.88 & 4.59 & 0.08 \\
\hline \multicolumn{10}{|c|}{ RWC } \\
\hline \multicolumn{5}{|c|}{ NIRONE S1.7 1350-1650 nm } & \multicolumn{5}{|c|}{ NIRONE S2.5 2000-2450 nm } \\
\hline & mean & $\max$ & $\min$ & std & & mean & $\max$ & $\min$ & std \\
\hline$R^{2}$ & 0.77 & 0.80 & 0.73 & 0.02 & $R^{2}$ & 0.81 & 0.82 & 0.79 & 0.007 \\
\hline RMSE & 6.86 & 7.47 & 6.47 & 0.32 & RMSE & 6.34 & 6.51 & 6.09 & 0.12 \\
\hline NRMSE & $9.3 \%$ & $10.1 \%$ & $8.8 \%$ & $0.4 \%$ & NRMSE & $8.6 \%$ & $8.8 \%$ & $8.3 \%$ & $0.2 \%$ \\
\hline MAE & 5.76 & 6.14 & 5.47 & 0.23 & MAE & 5.39 & 5.53 & 5.17 & 0.10 \\
\hline
\end{tabular}

We found strong correlations between the measured NRI spectral features and RWC and EWT (Table 2). The reflectance of single wavelengths explained from $66 \%$ to up to $87 \%$ of the variation in EWT and from $73 \%$ to $82 \%$ of the variation in RWC. Both sensors provided accurate estimates of RWC and EWT, but the 2000-2450 nm spectral region showed slightly higher accuracy in estimating both EWT and RWC.

The regression models that utilized two wavelengths (i.e., normalized ratio indices) were superior in terms of explaining variation in both EWT and RWC. The NRIs explained up to $96 \%$ and $93 \%$ of the variation in EWT using the 1350-1650 $\mathrm{nm}$ and $2000-2450 \mathrm{~nm}$ spectral regions, respectively. Variation in RWC was explained up to $90 \%$ and $89 \%$ by the $2000-2450$ $\mathrm{nm}$ and 1350-1650 nm spectral regions, respectively. The highest estimation accuracy of EWT was achieved with an NRI of $1390 \mathrm{~nm}$ and $1370 \mathrm{~nm}$ wavelengths providing an RMSE of $3.41 \mathrm{~g} / \mathrm{m}^{2}$. The highest estimation accuracy of RWC was achieved with NRI of wavelengths $2160 \mathrm{~nm}$ and $2090 \mathrm{~nm}$ providing an RMSE of $4.54 \%$-units. 
Table 3. Coefficient of determination $\left(R^{2}\right)$, root-mean-square-error (RMSE) and mean absolute error (MAE) for top five linear regression models for estimating equivalent water thickness $\left(E W T, \mathrm{~g} / \mathrm{m}^{2}\right.$ ) and relative water content (RWC, \%) using normalized ratio index (NRI) features $(n=44) . \gamma_{1}$ and $\gamma_{2}$ are the reflectance of each wavelength measured by NIRONE S1.7 and NIRONE S2.5 sensors and further used to derive NRI features.

EWT

\begin{tabular}{|c|c|c|c|c|c|c|c|c|c|}
\hline \multicolumn{5}{|c|}{ NIRONE S1.7 1350-1650 nm } & \multicolumn{5}{|c|}{ NIRONE S2.5 2000-2450 nm } \\
\hline$\lambda_{1}$ & $\lambda_{2}$ & RMSE & $R^{2}$ & MAE & $\lambda_{1}$ & $\lambda_{2}$ & RMSE & $R^{2}$ & MAE \\
\hline 1390 & 1370 & 3.41 & 0.96 & 2.66 & 2110 & 2020 & 4.32 & 0.93 & 3.52 \\
\hline 1390 & 1360 & 3.42 & 0.96 & 2.60 & 2110 & 2010 & 4.36 & 0.93 & 3.43 \\
\hline 1380 & 1370 & 3.42 & 0.96 & 2.68 & 2160 & 2020 & 4.36 & 0.93 & 3.50 \\
\hline 1390 & 1350 & 3.46 & 0.96 & 2.63 & 2110 & 2040 & 4.39 & 0.93 & 3.32 \\
\hline 1380 & 1360 & 3.48 & 0.95 & 2.69 & 2240 & 2020 & 4.39 & 0.93 & 3.49 \\
\hline \multicolumn{10}{|c|}{ RWC } \\
\hline \multicolumn{5}{|c|}{ NIRONE S1.7 1350-1650 nm } & \multicolumn{5}{|c|}{ NIRONE S2.5 2000-2450 nm } \\
\hline$\lambda_{1}$ & $\lambda 2$ & RMSE & $R^{2}$ & MAE & $\lambda_{1}$ & $\lambda 2$ & RMSE & $R^{2}$ & MAE \\
\hline 1620 & 1410 & 4.85 & 0.89 & 3.95 & 2160 & 2090 & 4.54 & 0.90 & 3.88 \\
\hline 1610 & 1410 & 4.86 & 0.89 & 3.93 & 2160 & 2040 & 4.65 & 0.90 & 3.90 \\
\hline 1650 & 1410 & 4.86 & 0.89 & 4.02 & 2160 & 2070 & 4.70 & 0.89 & 4.06 \\
\hline 1640 & 1410 & 4.87 & 0.89 & 4.02 & 2160 & 2080 & 4.75 & 0.89 & 4.05 \\
\hline 1630 & 1410 & 4.87 & 0.88 & 3.99 & 2160 & 2050 & 4.78 & 0.89 & 4.02 \\
\hline
\end{tabular}



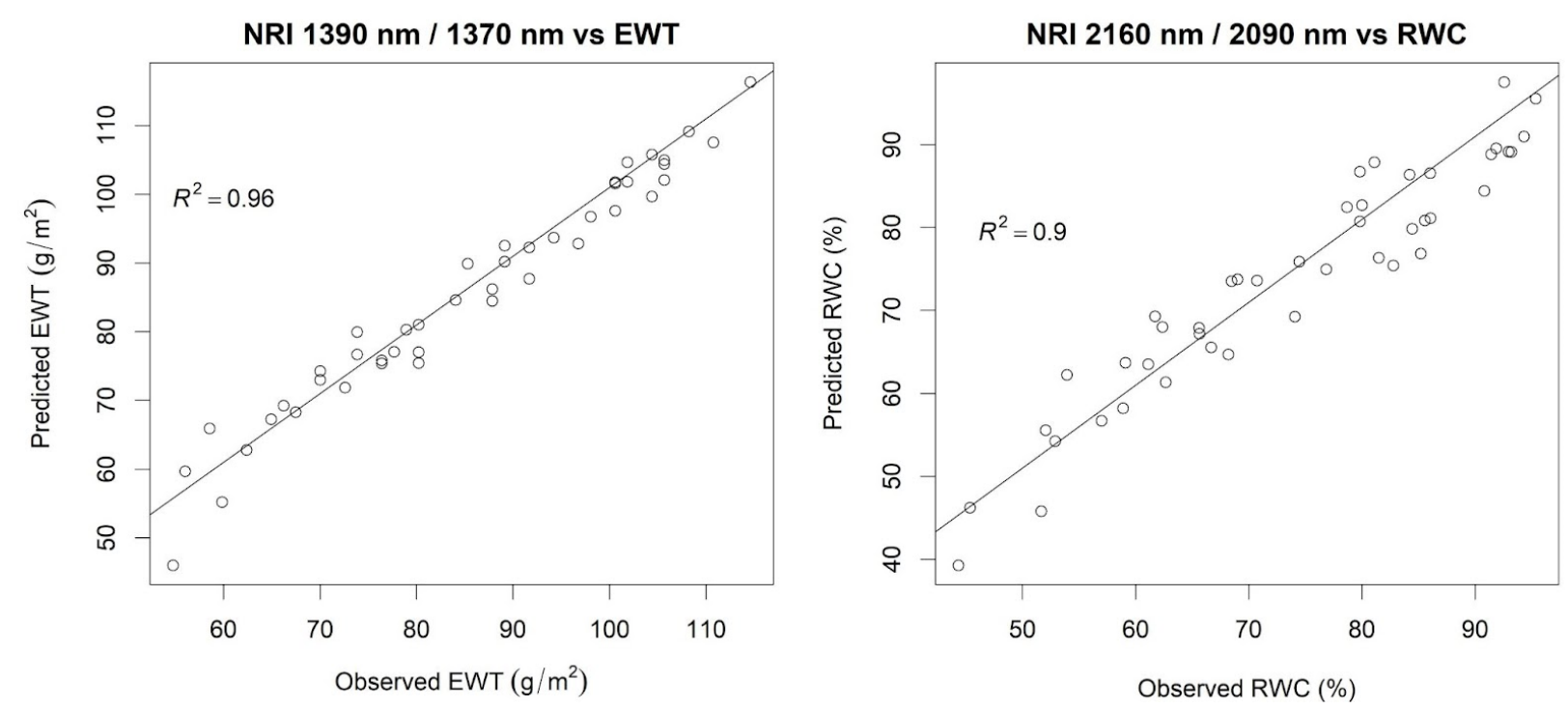

Figure 1. Observed and predicted equivalent water thickness (EWT) and relative water content (RWC) using the regression models with normalized ratio index (NRI) features that resulted in the highest accuracy based on coefficient of determination $\left(R^{2}\right)$ and root-meansquare-error. EWT was predicted using the NRI of $1390 \mathrm{~nm}$ and $1370 \mathrm{~nm}$ wavelengths and RWC was predicted using the NRI of $2160 \mathrm{~nm}$ and $2090 \mathrm{~nm}$ wavelengths.

\section{Time-series of hyperspectral imaging can capture diurnal leaf water dynamics}

\section{(Experiment 2)}

We used regression modelling to evaluate the capability of repeated hyperspectral measurements in monitoring leaf water content using the NRIs for predicting RWC. The used NRIs were identified to be most suitable in the laboratory measurements (Experiment 1). The fitted models of each leaf showed a consistent trend and were capable of capturing the changes in leaf water content (Table 3). The used NRIs that were identified as top indices in predicting RWC showed a strong correlation explaining $67-84 \%$ of the variation in xylem diameter. However, this was true only for the NIRONE S1.7 measuring the 1350-1650 nm spectral region. The $2000-2450 \mathrm{~nm}$ spectral region explained only $34-39 \%$ of the variation in xylem diameter.

Table 4. Mean, minimum (min), maximum ( $\max$ ) and standard deviation (std) of the coefficient of determinations $\left(R^{2}\right)$ for linear regression models between the 90 min lagged change in xylem diameter and normalized ratio indices (NRI) of wavelengths $\lambda_{1}$ and $\lambda_{2}$ for each monitored leaf $(n=10)$. The wavelengths were selected based on the results of the experiment 1 on the relation between the NRIs and relative water content. 


\begin{tabular}{ll|llll|ll|llll}
$\lambda_{1}(\mathbf{n m})$ & $\lambda_{2}(\mathbf{n m})$ & mean & $\mathbf{m i n}$ & $\mathbf{m a x}$ & $\mathbf{s t d}$ & $\lambda_{1}(\mathbf{n m})$ & $\lambda_{2}(\mathbf{n m})$ & mean & $\mathbf{m i n}$ & $\mathbf{m a x}$ & std \\
\hline $\mathbf{1 6 1 0}$ & $\mathbf{1 4 1 0}$ & 0.84 & 0.62 & 0.99 & 0.12 & $\mathbf{2 1 6 0}$ & $\mathbf{2 0 4 0}$ & 0.39 & 0.05 & 0.79 & 0.3 \\
$\mathbf{1 6 2 0}$ & $\mathbf{1 4 1 0}$ & 0.81 & 0.59 & 0.98 & 0.12 & $\mathbf{2 1 6 0}$ & $\mathbf{2 0 5 0}$ & 0.38 & 0.01 & 0.80 & 0.30 \\
$\mathbf{1 6 3 0}$ & $\mathbf{1 4 1 0}$ & 0.77 & 0.53 & 0.95 & 0.14 & $\mathbf{2 1 6 0}$ & $\mathbf{2 0 7 0}$ & 0.38 & 0.01 & 0.74 & 0.25 \\
$\mathbf{1 6 4 0}$ & $\mathbf{1 4 1 0}$ & 0.72 & 0.47 & 0.90 & 0.15 & $\mathbf{2 1 6 0}$ & $\mathbf{2 0 9 0}$ & 0.37 & 0.01 & 0.72 & 0.28 \\
$\mathbf{1 6 5 0}$ & $\mathbf{1 4 1 0}$ & 0.67 & 0.44 & 0.85 & 0.15 & $\mathbf{2 1 6 0}$ & $\mathbf{2 0 8 0}$ & 0.34 & 0.00 & 0.78 & 0.28
\end{tabular}

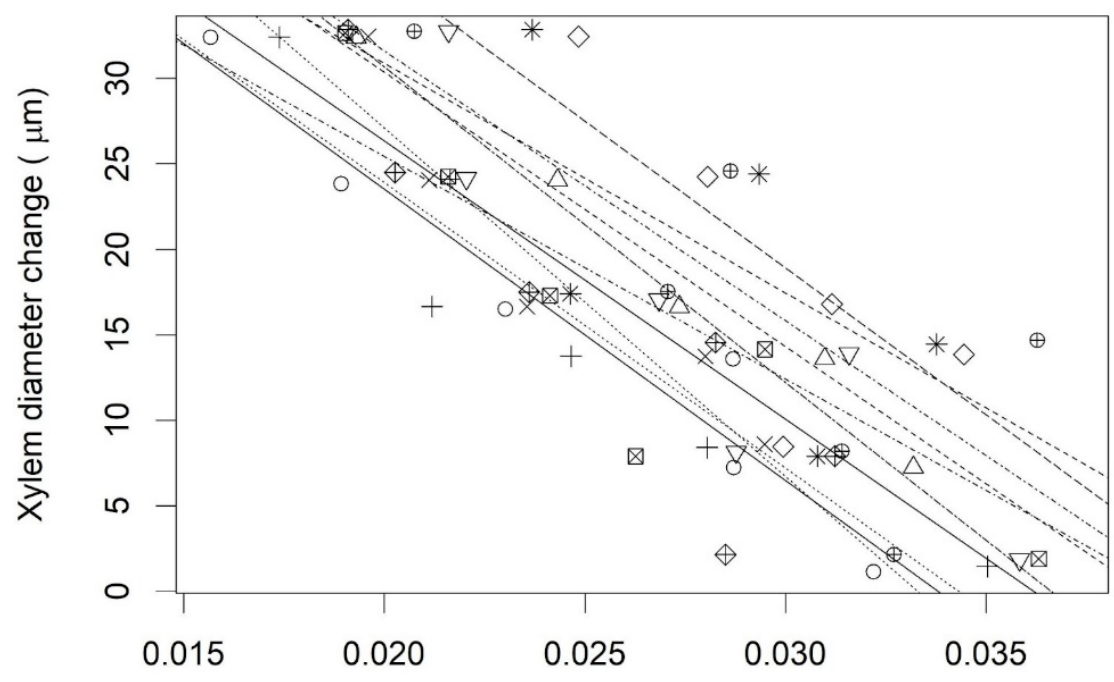

NRI $1620 \mathrm{~nm} / 1410 \mathrm{~nm}$

Figure 2. The normalized ratio index (NRI) of wavelengths $1620 \mathrm{~nm}$ and $1420 \mathrm{~nm}$ for each leaf $(n=10)$ plotted against change in xylem diameter. The lines represent fitted regression models between the NRI and change in diameter. Plot symbols represent each leaf sample. Xylem diameter is expressed in relation to xylem diameter at the smallest diameter measured during the measurement period.

We developed regression models between all the leaf measurements and the change in xylem diameter and found that NRI of several wavelengths were able to explain $79 \%$ of the variation in xylem diameter (Table 4). The wavelengths differed from the best predictors of leaf water content identified in the laboratory measurements (Experiment 1). 
Table 5. Summary of coefficient of determination $\left(R^{2}\right)$ for linear regression models between normalized ratio indices, calculated using $\gamma_{1}$ and $\gamma_{2}$ that are the reflectance of each wavelength, and the change in stem diameter for all leaf samples pooled together $(n=60)$.

\begin{tabular}{cc|c}
$\lambda_{1}$ & $\lambda_{2}$ & $\boldsymbol{R}^{2}$ \\
\hline 1530 & 1440 & 0.79 \\
1540 & 1430 & 0.78 \\
1550 & 1430 & 0.78 \\
1520 & 1450 & 0.78 \\
1500 & 1460 & 0.78
\end{tabular}

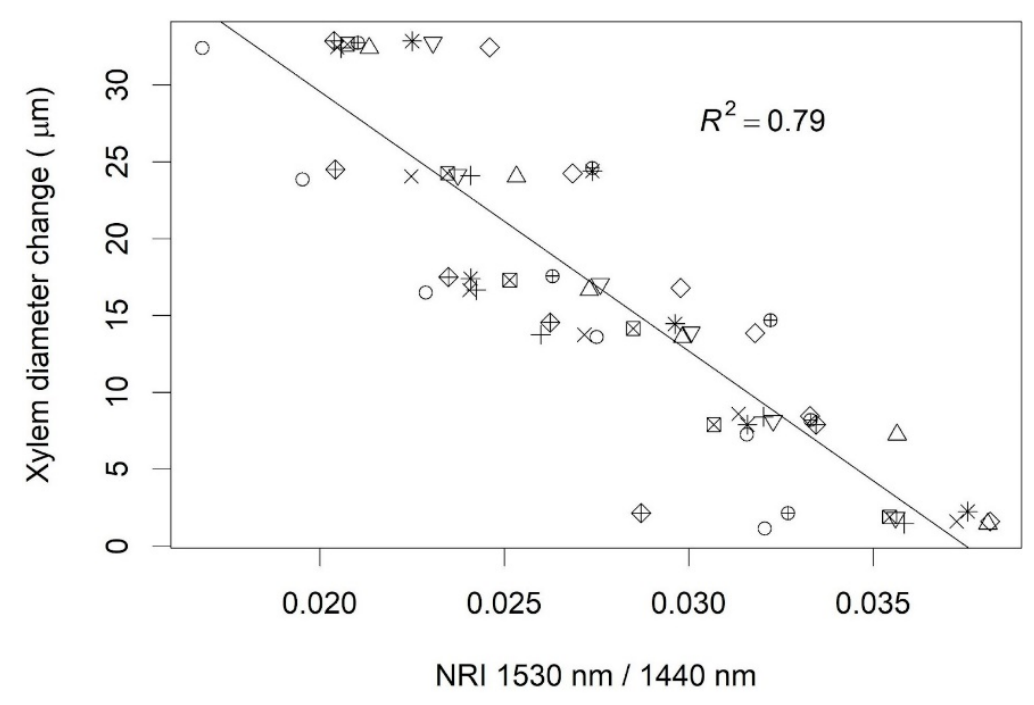

Figure 3. The relationship between a normalized ratio index (NRI) of $1530 \mathrm{~nm}$ and $1440 \mathrm{~nm}$ wavelengths and change in xylem diameter. Each symbol denotes a different leaf.

In-situ monitoring reveals detailed diurnal leaf water content dynamics (Experiment 3)

Monitoring of single $D$. marginate leaves revealed diurnal dynamics in the leaf reflectance. The dynamics were observable at several spectral features in the spectral region from $1350 \mathrm{~nm}$ to $1950 \mathrm{~nm}$ (Figures $4 \& 5$ ). NIRONE S2.5 sensor, which utilizes the $2000 \mathrm{~nm}$ to $2450 \mathrm{~nm}$ spectral 
region, did not exhibit a strong diurnal dynamic (Figure 6). It could be observed that the diurnal variation of the reflectance signal was affected by both air temperature as air changes in air temperature mainly control the evaporative demand due to its effect on VPD, and time from last watering. The amplitude of spectral variation was greater in well-watered conditions and reduced as time from last watering increased (Figures $4 \& 5$ ).

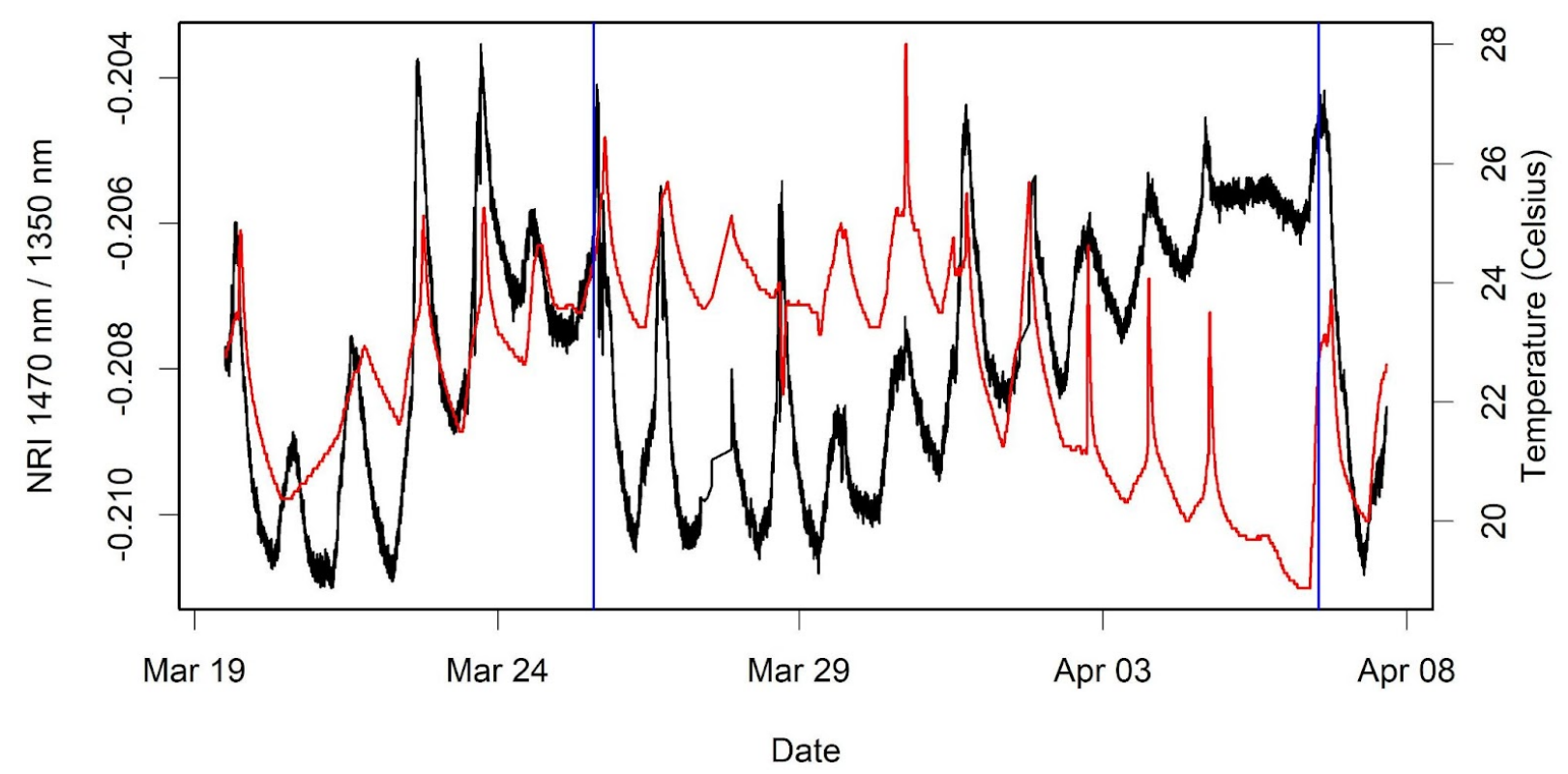

Figure 4. The moving average of 10 reflectance measurements of normalized ratio index (NRI) of $1470 \mathrm{~nm}$ and $1350 \mathrm{~nm}$ wavelengths (black line) and air temperature (red line) during the monitoring period (March 19-April 8) of a Dracaena marginate (Lem.) leaf. Blue vertical lines denote a watering. The reflectance measurements were conducted every 48 seconds and temperature measurements every 15 minutes.

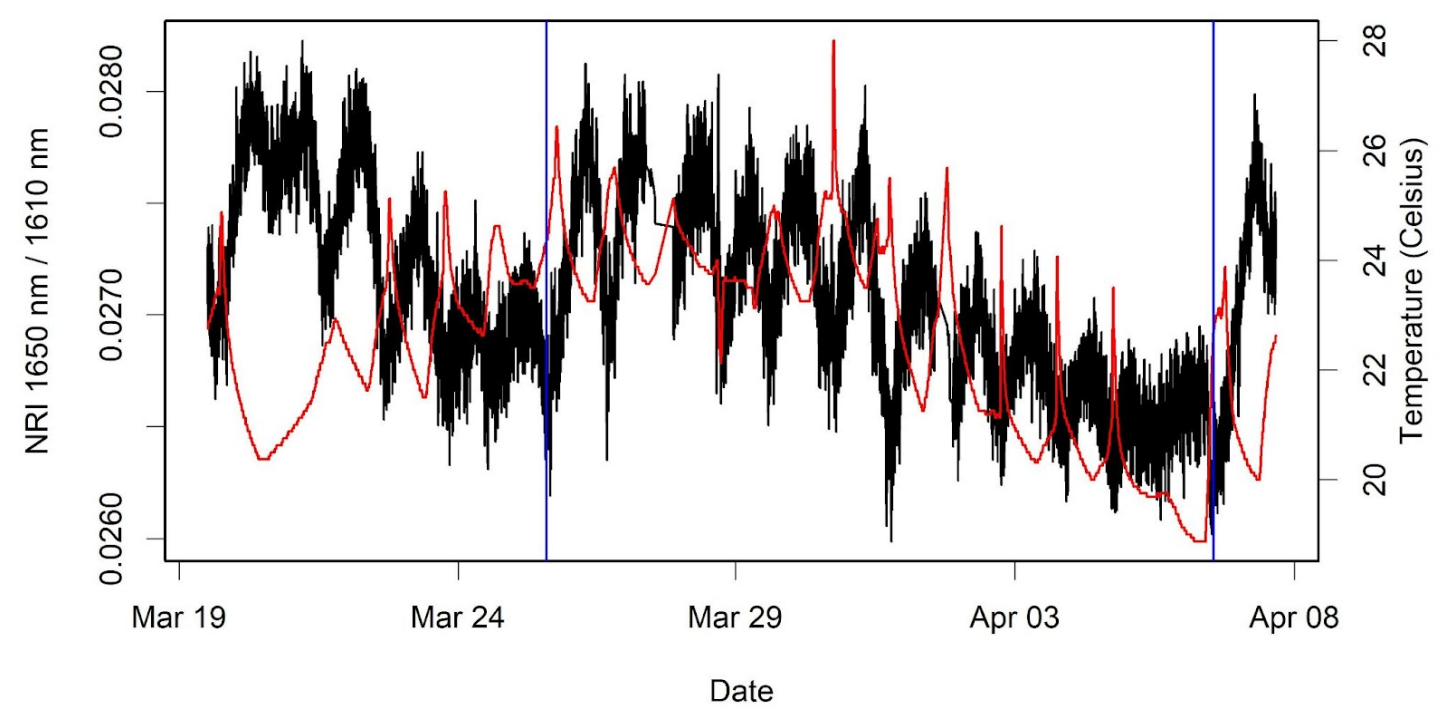


Figure 5. Moving average of 10 measurements of normalized ratio index (NRI) of $1650 \mathrm{~nm}$ and $1610 \mathrm{~nm}$ wavelengths (black line) of a Dracaena marginate (Lem.) leaf and air temperature (red line) during the monitoring period (March 19-April 8). The vertical blue lines denote the timing of watering. The reflectance measurements were conducted every 48 seconds and temperature measurements every 15 minutes.

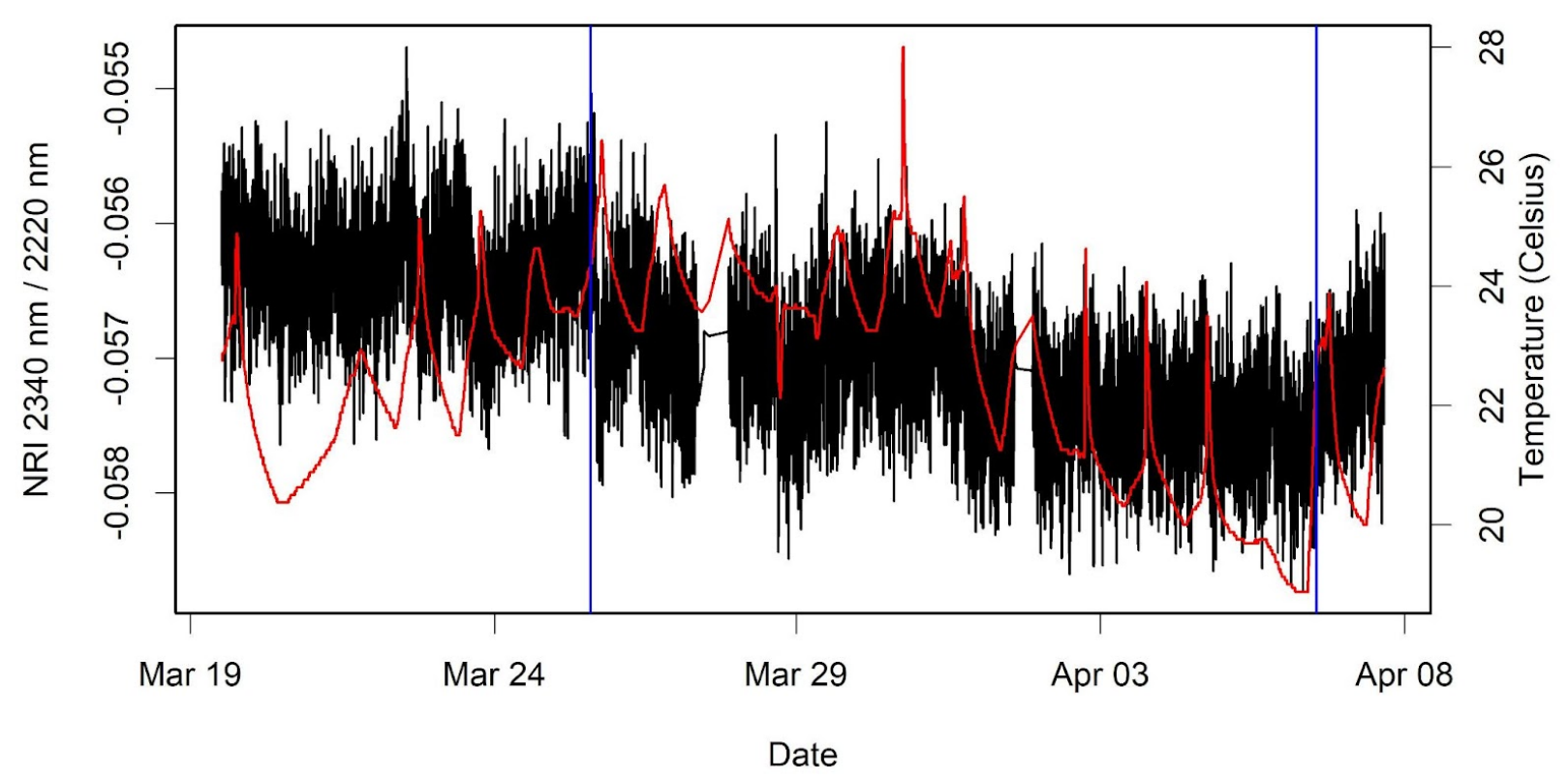

Figure 6. Moving average of 10 measurements of normalized ratio index (NRI) of $2340 \mathrm{~nm}$ and $2220 \mathrm{~nm}$ wavelengths (black line) of a Dracaena marginate (Lem.) leaf and air temperature (red line) during the monitoring period (March 19-April 8). The vertical blue lines denote the timing of watering. The reflectance measurements were conducted every 48 seconds and temperature measurements every 15 minutes.

The NIRONE S1.7, S2.0 and S2.5 sensors showed to be able to explain most variation in TLW ranging from $91 \%$ using single wavelengths to $99 \%$ using NRIs (Table 5) reflecting the reduction of leaf water content as drought increased. NRIs outperformed reflectance of single wavelengths in terms of $R^{2}$. The mean of NRI using $2340 \mathrm{~nm}$ and $2220 \mathrm{~nm}$ wavelengths showed nearly 1:1 correlation with TLW, but it could be observed that the measurements of NIRONE S2.5 using the 2000-2450 nm spectral range contained much more noise than the other sensors reducing the ability to measure diurnal dynamics of leaf water content (Figure 6).

Table 6. Coefficient of determination $\left(R^{2}\right)$ for the top five regression models between time from last watering (TLW) and daily metrics of spectral features for each sensor. The 
monitoring period lasted 12 days. $\gamma_{1}$ and $\gamma_{2}$ are the reflectance of each wavelength used in normalized ratio index calculations.

NIRONE S1.7 1350-1650 nm

\begin{tabular}{rrrc|rrr}
\hline & \multicolumn{3}{c|}{ NRI $\left(\lambda_{1}, \lambda_{2}\right)$} & & \multicolumn{3}{c}{ Reflectance at $\lambda$} \\
$\lambda_{1}$ & $\lambda_{2}$ & $\boldsymbol{R}^{2}$ & feature & $\lambda$ & $\boldsymbol{R}^{2}$ & feature \\
\hline $\mathbf{1 5 4 0}$ & 1530 & 0.91 & mean & 1460 & 0.76 & range \\
$\mathbf{1 4 2 0}$ & 1400 & 0.90 & mean & 1450 & 0.75 & range \\
$\mathbf{1 4 1 0}$ & 1400 & 0.88 & mean & 1470 & 0.74 & range \\
$\mathbf{1 4 7 0}$ & 1350 & 0.87 & mean & 1370 & 0.74 & range \\
$\mathbf{1 5 5 0}$ & 1530 & 0.87 & mean & 1360 & 0.72 & range \\
\hline
\end{tabular}

NIRONE S2.0 1550-1950 nm

\begin{tabular}{lccc|ccc}
\hline \multicolumn{5}{c|}{ NRI $\left(\lambda_{1}, \lambda_{2}\right)$} & & \multicolumn{3}{c}{ Reflectance at $\lambda$} \\
$\lambda_{1}$ & $\lambda_{2}$ & $\boldsymbol{R}^{2}$ & feature & $\lambda$ & $\boldsymbol{R}^{2}$ & feature \\
\hline $\mathbf{1 6 5 0}$ & 1610 & 0.96 & mean & $\mathbf{1 5 6 0}$ & 0.53 & range \\
$\mathbf{1 6 4 0}$ & 1610 & 0.95 & mean & $\mathbf{1 5 5 0}$ & 0.52 & range \\
$\mathbf{1 6 5 0}$ & 1620 & 0.95 & mean & $\mathbf{1 5 7 0}$ & 0.51 & range \\
$\mathbf{1 6 6 0}$ & 1610 & 0.95 & mean & $\mathbf{1 5 8 0}$ & 0.50 & range \\
$\mathbf{1 6 4 0}$ & 1620 & 0.95 & mean & $\mathbf{1 5 9 0}$ & 0.49 & range \\
\hline
\end{tabular}

NIRONE S2.5 2000-2450 nm

\begin{tabular}{llll|lll}
\hline & & & & \multicolumn{2}{c}{ Reflectance at $\lambda$} \\
$\lambda_{1}$ & $\lambda_{2}$ & $R^{2}$ & feature & $\lambda$ & $R^{2}$ & feature \\
\hline
\end{tabular}




\begin{tabular}{llll|lll}
\hline $\mathbf{2 3 4 0}$ & $\mathbf{2 2 2 0}$ & 0.99 & mean & $\mathbf{2 4 5 0}$ & 0.90 & mean \\
$\mathbf{2 3 3 0}$ & $\mathbf{2 2 6 0}$ & 0.99 & mean & $\mathbf{2 4 4 0}$ & 0.88 & mean \\
$\mathbf{2 3 4 0}$ & $\mathbf{2 3 1 0}$ & 0.99 & range & $\mathbf{2 4 3 0}$ & 0.85 & mean \\
$\mathbf{2 3 3 0}$ & $\mathbf{2 2 7 0}$ & 0.99 & mean & $\mathbf{2 4 2 0}$ & 0.83 & mean \\
$\mathbf{2 3 2 0}$ & $\mathbf{2 2 6 0}$ & 0.99 & mean & $\mathbf{2 4 1 0}$ & 0.80 & mean \\
\hline
\end{tabular}

\section{Discussion}

In this study, we aimed to demonstrate the capabilities of hyperspectral spectroscopy in measuring timely changes in leaf water content. We showed that low-cost hyperspectral sensors can be used to monitor changes in leaf water content that occur in a diurnal fashion.

We found strong linear relationships between the measured spectra, the spectral features, and leaf water content measured both as EWT and RWC, in our laboratory experiment (Experiment 1). A slightly higher accuracy was achieved in estimating EWT than RWC and there were differences in the wavelengths that provided these estimates. EWT was estimated best with a narrow spectral region using spectral features between $1350 \mathrm{~nm}$ and $1390 \mathrm{~nm}$, but longer wavelengths resulted in the most reliable RWC estimates with an NRI of $1620 \mathrm{~nm}$ and 1410 $\mathrm{nm}$ and NRI of $2160 \mathrm{~nm}$ and $2090 \mathrm{~nm}$ wavelengths. Similar results have been obtained many times before in several studies, showcasing various techniques that can be used to estimate leaf water content (Cheng et al., 2011; Feret et al., 2011). The purpose of the first experiment was to validate the well-known relationships between leaf water content and the spectral features and to give an estimate of the accuracy of the used method in estimating EWT and RWC.

The purpose of the second experiment was to test the sensors in a forest field experiment with mature trees and measure diurnal dynamics of leaf water content. We found that the spectral features were in good agreement with the diurnal variation of xylem diameter, which is known to be well-correlated with leaf water dynamics generally (Offenthaler et al., 2001) and also at the same test site (Junttila et al., 2021). We found that the change in spectral features correlated well with the change in xylem diameter, but the absolute values of the spectral features varied between leaves. This indicates that there are other leaf structural components, such as specific 
leaf area, that are influencing the spectra, complicating the direct estimation of RWC or EWT (Feret et al., 2011). Some spectral features also gave reasonable agreement with xylem diameter variation with all the leaves pooled in the same model, indicating that a more general model could be achievable. The changes in xylem diameter integrate the changes in leaf water content over the entire canopy, but the actual leaf water content varies between leaves due to differences in local light environment and microclimate.

The measurements of the second experiment were conducted using only single measurements aiming at avoiding the change of exact measurement position between different measurements. This could have affected the results, because of possible variation in the measurements and it could be more advisable to use many measurements of a single leaf. Afterall, the measurement area of the NIRONE sensor is tiny, about a millimetre in diameter, and the viewing angle is very narrow, only about 8 degrees. Therefore, multiple measurements of each leaf could give a more stable measurement of the leaf spectra, which can be influenced also by varying leaf water content within the leaf.

The third experiment demonstrated continuous in-situ monitoring of leaf spectra and leaf water content. The diurnal dynamics of leaf water content were clearly observable in the leaf spectra with spectral features from both the 1350-1650 nm region (NIRONE S1.7) and the 1550-1950 $\mathrm{nm}$ region (NIRONE S2.0) (Figures 4 and 5), but the 2000-2450 $\mathrm{nm}$ spectral region (NIRONE S2.5) did not show a clear diurnal trend due to a high amount of noise in the measurement (Figure 6). It can be observed from the figures how the amplitude of diurnal leaf water content decreases as drought develops and is restored after the plant is watered. The signal-to-noise ratio of the NIRONE S2.5 sensor seemed to hamper the measurement of diurnal dynamics despite the high correlation between TLW and spectral features and the high sensitivity to leaf water content (Feret et al., 2019). Therefore, the NIRONE sensors S1.7 and S2.0 seem like more viable options for continuous measurement of leaf water content.

The daily dynamics that were observed in the $D$. marginate leaves correspond well with previous measurements of diurnal sap flow and xylem diameter dynamics in drought conditions (Jupa et al., 2017). We could observe in our measurements that the amplitude of variation was decreasing towards more severe drought conditions and that the amplitude of variation returned after watering similarly to the observations made by Jupa et al. (2017). Although it is challenging to fully understand the magnitude of changes in water content and convert the observed values in reflectance to absolute amounts of water, we can use this type of data to 
understand the variability of leaf water content. There is also a vast body of literature that supports our findings of the sensitivity of the investigated spectral regions on leaf water content (Cheng et al., 2011; Feret et al., 2008; Romer et al., 2012). We already have a detailed understanding of the physical changes of water on electromagnetic radiation and the relationship between water content and short-wave infrared radiation (Becker and Autler, 1946; Lunkenheimer et al., 2017), but the availability of suitable instruments has been lacking the full application of this knowledge.

We observed that the measurement setup is very sensitive to any changes in viewing angle and distance on the measured spectra (not reported). Therefore, we suggest to measure the daily changes of leaf water content, the sensor needs to be carefully installed and care taken so that the leaf does not change position during the measurement period and does not cause alteration to the measured reflectance due to changes in bidirectional reflectance distribution function.

A disadvantage of the presented method is the lack of multiple pixels compared to other imaging sensors, such as terahertz radiation spectroscopy (Browne et al., 2020) or terrestrial lidar (Elsherif et al., 2019b; Junttila et al., 2021). Spatial variation of leaf water content is more difficult to measure using the presented method, but on the other hand we were able to observe very clearly the small diurnal variation in leaf water content, which has been difficult to measure non-destructively. An interesting future approach would be to combine a time-series of hyperspectral spectroscopy at leaf-level and use that to up-scale the measurements to canopylevel using terrestrial lidar.

The results of this study showcase how low-cost hyperspectral spectroscopy can be used to estimate EWT and RWC and assess detailed leaf water content dynamics through time. The NIRONE sensors seem to embody spectral resolution and accuracy that enables the monitoring of leaf spectra in the short-wave infrared region at a reasonable cost allowing multiple applications of monitoring leaf water content. Detailed temporal monitoring of leaf water dynamics can help us to further understand the movement of water within the soil-treeatmosphere continuum. These low-cost sensors open new avenues for research in studying plant-water interactions by allowing continuous measurements and the detection of minute changes in leaf water content. 


\section{Acknowledgements}

The research was funded by the Academy of Finland [grant numbers 330422, 315079, 345166, 337656, 337811 and 337810]. This study has been done with affiliation to the Academy of Finland Flagship Forest-Human-Machine Interplay - Building Resilience, Redefining Value Networks and Enabling Meaningful Experiences (UNITE) [grant number 337127].

\section{References}

Allen, C.D., Macalady, A.K., Chenchouni, H., Bachelet, D., McDowell, N., Vennetier, M., Kitzberger, T., Rigling, A., Breshears, D.D., Hogg, E.H., Gonzalez, P., Fensham, R., Zhang, Z., Castro, J., Demidova, N., Lim, J.H., Allard, G., Running, S.W., Semerci, A., \& Cobb, N. (2010). A global overview of drought and heat-induced tree mortality reveals emerging climate change risks for forests. Forest Ecology and Management, 259, 660684.

Beć, K.B., Grabska, J., Siesler, H.W., \& Huck, C.W. (2020). Handheld near-infrared spectrometers: Where are we heading? NIR news, 31, 28-35.

Becker, G.E., \& Autler, S.H. (1946). Water vapor absorption of electromagnetic radiation in the centimeter wave-length range. Physical Review, 70, 300.

Browne, M., Yardimci, N.T., Scoffoni, C., Jarrahi, M., \& Sack, L. (2020). Prediction of leaf water potential and relative water content using terahertz radiation spectroscopy. Plant direct, 4, e00197.

Ceccato, P., Flasse, S., Tarantola, S., Jacquemoud, S., \& Gregoire, J.M. (2001). Detecting vegetation leaf water content using reflectance in the optical domain. Remote Sensing of Environment, 77, 22-33.

Cheng, T., Rivard, B., \& Sanchez-Azofeifa, A. (2011). Spectroscopic determination of leaf water content using continuous wavelet analysis. Remote Sensing of Environment, 115, 659-670.

Danson, F.M., Steven, M.D., Malthus, T.J., \& Clark, J.A. (1992). High-Spectral Resolution Data for Determining Leaf Water-Content. International Journal of Remote Sensing, 13, 461-470.

Egilla, J.N., Davies, F.T., \& Boutton, T.W. (2005). Drought stress influences leaf water content, photosynthesis, and water-use efficiency of Hibiscus rosa-sinensis at three potassium concentrations. Photosynthetica, 43, 135-140. 
Elsherif, A., Gaulton, R., \& Mills, J. (2019a). Four Dimensional Mapping of Vegetation Moisture Content Using Dual-Wavelength Terrestrial Laser Scanning. Remote Sensing, $11,2311$.

Elsherif, A., Gaulton, R., Shenkin, A., Malhi, Y., \& Mills, J. (2019b). Three dimensional mapping of forest canopy equivalent water thickness using dual-wavelength terrestrial laser scanning. Agricultural and Forest Meteorology, 276, 107627.

Feret, J.B., Francois, C., Asner, G.P., Gitelson, A.A., Martin, R.E., Bidel, L.P.R., Ustin, S.L., le Maire, G., \& Jacquemoud, S. (2008). PROSPECT-4 and 5: Advances in the leaf optical properties model separating photosynthetic pigments. Remote Sensing of Environment, $112,3030-3043$.

Feret, J.B., Francois, C., Gitelson, A., Asner, G.P., Barry, K.M., Panigada, C., Richardson, A.D., \& Jacquemoud, S. (2011). Optimizing spectral indices and chemometric analysis of leaf chemical properties using radiative transfer modeling. Remote Sensing of Environment, 115, 2742-2750.

Feret, J.B., le Maire, G., Jay, S., Berveiller, D., Bendoula, R., Hmimina, G., Cheraiet, A., Oliveira, J.C., Ponzoni, F.J., Solanki, T., de Boissieu, F., Chave, J., Nouvellon, Y., Porcar-Castell, A., Proisy, C., Soudani, K., Gastellu-Etchegorry, J.P., \& LefevreFonollosa, M.J. (2019). Estimating leaf mass per area and equivalent water thickness based on leaf optical properties: Potential and limitations of physical modeling and machine learning. Remote Sensing of Environment, 231, 110959.

Huck, C.W. (2021). New Trend in Instrumentation of NIR Spectroscopy-Miniaturization. Near-Infrared Spectroscopy (pp. 193-210): Springer.

Junttila, S., Holopainen, M., Vastaranta, M., Lyytikäinen-Saarenmaa, P., Kaartinen, H., Hyyppä, J., \& Hyyppä, H. (2019). The potential of dual-wavelength terrestrial lidar in early detection of Ips typographus (L.) infestation-Leaf water content as a proxy. Remote Sensing of Environment, 231, 111264.

Junttila, S., Hölttä, T., Puttonen, E., Katoh, M., Vastaranta, M., Kaartinen, H., Holopainen, M., \& Hyyppä, H. (2021). Terrestrial laser scanning intensity captures diurnal variation in leaf water potential. Remote Sensing of Environment, 255, 112274.

Jupa, R., Plichta, R., Paschová, Z., Nadezhdina, N., \& Gebauer, R. (2017). Mechanisms underlying the long-term survival of the monocot Dracaena marginata under drought conditions. Tree physiology, 37, 1182-1197. 
Konings, A.G., Rao, K., \& Steele-Dunne, S.C. (2019). Macro to micro: microwave remote sensing of plant water content for physiology and ecology. New Phytologist, 223, 11661172 .

Korpela, I. (2017). Acquisition and evaluation of radiometrically comparable multi-footprint airborne LiDAR data for forest remote sensing. Remote Sensing of Environment, 194, 414-423.

Kotz, B., Schaepman, M., Morsdorf, F., Bowyer, P., Itten, K., \& Allgower, B. (2004). Radiative transfer modeling within a heterogeneous canopy for estimation of forest fire fuel properties. Remote Sensing of Environment, 92, 332-344.

Li-Ping, B., Fang-Gong, S., Ti-Da, G., Zhao-Hui, S., Yin-Yan, L., \& Guang-Sheng, Z. (2006). Effect of soil drought stress on leaf water status, membrane permeability and enzymatic antioxidant system of maize. Pedosphere, 16, 326-332.

Lunkenheimer, P., Emmert, S., Gulich, R., Köhler, M., Wolf, M., Schwab, M., \& Loidl, A. (2017). Electromagnetic-radiation absorption by water. Physical Review E, 96, 062607.

Offenthaler, I., Hietz, P., \& Richter, H. (2001). Wood diameter indicates diurnal and longterm patterns of xylem water potential in Norway spruce. Trees, 15, 215-221.

Penuelas, J., Filella, I., Biel, C., Serrano, L., \& Save, R. (1993). The reflectance at the 950$970 \mathrm{~nm}$ region as an indicator of plant water status. International Journal of Remote Sensing, 14, 1887-1905.

Penuelas, J., Pinol, J., Ogaya, R., \& Filella, I. (1997). Estimation of plant water concentration by the reflectance water index WI (R900/R970). International Journal of Remote Sensing, 18, 2869-2875.

Rao, K., Anderegg, W.R.L., Sala, A., Martinez-Vilalta, J., \& Konings, A.G. (2019). Satellitebased vegetation optical depth as an indicator of drought-driven tree mortality. Remote Sensing of Environment, 227, 125-136.

Romer, C., Wahabzada, M., Ballvora, A., Pinto, F., Rossini, M., Panigada, C., Behmann, J., Leon, J., Thurau, C., Bauckhage, C., Kersting, K., Rascher, U., \& Plumer, L. (2012). Early drought stress detection in cereals: simplex volume maximisation for hyperspectral image analysis. Functional Plant Biology, 39, 878-890.

Tucker, C.J. (1980). Remote sensing of leaf water content in the near infrared. Remote Sensing of Environment, 10, 23-32.

Turner, N., \& Long, M. (1980). Errors arising from rapid water loss in the measurement of leaf water potential by the pressure chamber technique. Functional Plant Biology, 7, 527 537. 
White, D., Beadle, C., \& Worledge, D. (1996). Leaf water relations of Eucalyptus globulus ssp. globulus and E. nitens: seasonal, drought and species effects. Tree physiology, 16, 469-476.

Williams, A.P., Allen, C.D., Macalady, A.K., Griffin, D., Woodhouse, C.A., Meko, D.M., Swetnam, T.W., Rauscher, S.A., Seager, R., Grissino-Mayer, H.D., Dean, J.S., Cook, E.R., Gangodagamage, C., Cai, M., \& McDowell, N.G. (2013). Temperature as a potent driver of regional forest drought stress and tree mortality. Nature climate change, 3, 292297. 